\title{
NOTA
}

\section{COBERTURA DO SOLO E ESTOQUE DE NUTRIENTES DE DUAS LEGUMINOSAS PERENES, CONSIDERANDO ESPAÇAMENTOS E DENSIDADES DE PLANTIO(1)}

\author{
A. PERIN(2), J . G. M. GUERRA(3), M. G. TEIXEIRA ${ }^{(3)}$ \& E. ZONTA(4)
}

\begin{abstract}
RESUMO
O estabelecimento de leguminosas herbáceas perenes nos sistemas de produção constitui ainda um desafio, principalmente por apresentarem crescimento inicial lento. Para viabilizar sua implantação, este trabalho objetivou determinar as taxas de cobertura do solo, produção de matéria seca, teores e acumulação de N, P e K das leguminosas herbáceas perenes galáxia (Galactia striata) e cudzu tropical (Pueraria phaseoloides), considerando espaçamentos e densidades de plantio. 0 experimento, instalado em dezembro/ 98 na E mbrapa Agrobiologia, Seropédica (RJ ), constou do delineamento em blocos ao acaso, em arranjo fatorial $2 \times 2 \times 4$, com quatro repetições. Os tratamentos constaram das espécies galáxia e cudzu tropical, plantadas em dois espaçamentos entre sulcos de plantio $(25$ e $50 \mathrm{~cm})$ e quatro densidades de plantas $(5,10,15$ e 20 plantas $\mathrm{m}^{-1}$ ). A densidade adequada para a rápida cobertura do solo para cudzu tropical e galáxia foi de 10 plantas $\mathrm{m}^{-1}$, no espaçamento de $25 \mathrm{~cm}$ entre os sulcos de plantio. A maior produção de matéria seca e acumulação de N, P e K na parte aérea das plantas foram evidenciadas apenas no primeiro corte, sendo os maiores valores obtidos no espaçamento de $25 \mathrm{~cm}$ e na densidade de 10 plantas $\mathrm{m}^{-1}$. 0 espaçamento de $25 \mathrm{~cm}$ com 10 plantas $\mathrm{m}^{-1}$ foi a combinação mais adequada para a plena formação da cobertura viva do solo com cudzu tropical e galáxia.
\end{abstract}

Termos de indexação: arranjo populacional, plantas de cobertura, reciclagem de nutrientes, Galactia striata, Pueraria phaseoloides.

\footnotetext{
(1) Trabal ho executado com recursos da Embrapa - Centro Nacional de Pesquisa de Agrobiologia subprojeto no 01.096.032-08, CEP: 23890-000, Seropédica - RJ . Recebido para publicação em março de 2003 e aprovado em novembro de 2003.

(2) Doutorando em Fitotecnia, Departamento de Fitotecnia, Universidade Federal de Viçosa - UFV. CEP 36571-000 Viçosa, MG. E-mail: aperin@vicosa.ufv.br

(3) Pesquisador da Embrapa, Centro Nacional de Pesquisa de Agrobiologia. Caixa Postal 74505, CEP 23890-000 Seropédica (RJ). E-mails: gmguerra@cnpab.embrapa.br; grandi@cnpab.embrapa.br

(4) Professor do Departamento de Solos, Universidade Federal Rural do Rio de J aneiro - UFRRJ. CEP 23890-000 Seropédica (RJ ). E-mail: zonta@ufrrj.br
} 


\title{
SUMMARY: SOIL COVER AND NUTRIENT ACCUMULATION OF TWO PERENNIAL LEGUMES AS FUNCTIONS OF SPACING AND PLANTING DENSITIES
}

\begin{abstract}
Theestablishment of herbaceous perennial leguminous in production systems is still a challenge, mainly because of thesl ow initial growth. Evaluations of thesoil cover ratio, dry matter production, contents and accumulati ons of nitrogen, phosphorus, and potassium of the herbaceous perennial Galactia striata and Pueraria phaseoloides, sown in different densities and row spacing, ai med at making their establ ishments feasi ble. Theexperiment was conducted in December/ 98 at Embrapa Agrobi ol ogia, Seropédica (RJ ), in a completely randomized block design, as a factorial arrangement $2 \times 2 \times 4$, with four replications. The treatments consisted of the plant species Galactia striata and Pueraria phaseol oi des, planted in two spacings ( 25 and $50 \mathrm{~cm}$ apart) and four sowing densities (5, 10, 15 and 20 plants $\mathrm{m}^{-1}$ ). Themost adequatedensity for a fast soil cover was 10 plants $\mathrm{m}^{-1}$ for Pueraria phaseol oides and Galactia striata, in a $25 \mathrm{~cm}$ spacing between planting rows. The highest dry matter production and accumulation of N,P and K in theaerial part of the plant werefound in the first cut, in a spacing of $25 \mathrm{~cm}$ and row density of 10 plants $\mathrm{m}^{-1}$. The $25 \mathrm{~cm}$ spacing with 10 plants $\mathrm{m}^{-1}$ was identified as the most adequate combination for the formation of a full soil cover with Pueraria phaseoloides and Galactia striata.
\end{abstract}

Index terms: population arrangement, soil cover crop, nutrient cycling, Galactia striata, Pueraria phaseoloides.

\section{INTRODUÇÃO}

Práticas de manejo e conservação, como o emprego de plantas de cobertura, são rel evantes para a manutenção ou melhoria das características químicas, físicas e biológicas dos solos. A adubação verde consiste no emprego de espécies de diferentes famílias botânicas, nativas ou introduzidas, que cobrem o terreno em períodos de tempo ou durante todo ano. Destacam-se as espécies pertencentes à família das leguminosas, por formarem associações simbióticas com bactérias fixadoras de $\mathrm{N}_{2}$, o que resulta no aporte de quantidades expressivas desse nutriente ao solo.

Apesar dos esforços para ampliar o uso da adubação verde, as ações de pesquisa visaram, principalmente, à geração de base técnico-científica para o emprego de leguminosas anuais. No entanto, há um espectro de espécies herbáceas de ciclo perene, com grande potencial de utilização como cobertura viva permanente de solo, notadamente em pomares. Estas plantas, além de protegerem o solo dos agentes climáticos, seqüestram $\mathrm{C}$ e fixam $\mathrm{N}$ atmosférico e, desta forma, apresentam potencial para manter ou el evar oteor de matéria orgânica, mobilizar ereciclar nutrientes e favorecer a atividade biológica do solo (Guerra \& Teixeira, 1997; Perin, 2001; Duda et al., 2003). Contudo, a identificação e a adequação desse grupo de leguminosas nos sistemas de produção ainda constituem um desafio.

Além disso, as leguminosas perenes competem com espécies espontâneas e interferem no ciclo reprodutivo dessas espécies, o que reduz a mão-deobra empregada no controle da vegetação espontânea, como observado por Wiles et al. (1989), Lanini et al. (1989) eSarrantonio (1992). No cultivo de hortaliças, Kleinhenz et al. (1997) constataram que o uso das leguminosas perenes centrosema (Centrosema pubescens), siratro (Macroptilium atropurpureum) e desmódio (Desmodium intortum) promoveu o controle das espécies espontâneas, não prejudicou o desempenho das hortal iças e forneceu $\mathrm{N}$ às culturas.

Apesar dos benefícios, um entrave na aplicação desta prática está noestabel eaimento das leguminosas perenes para a formação da cobertura viva do solo. Segundo Perin et al. (2000), um aspecto importante no estabelecimento da cobertura viva é a taxa de crescimento das leguminosas perenes, inicial mente lenta, quando comparada à de leguminosas anuais. Desta forma, são necessários cuidados que assegurem a supressão da vegetação espontânea, atéqueas plantas se estabel eçam. Por isso, osucesso dessa prática depende, dentre outros fatores, da taxa decrescimento dessas plantas, de forma que possam competir efetivamente com a vegetação espontânea.

Trabal hos que relacionam arranjos populacionais edesempenhodeleguminosas anuais têm evidenciado a importância da densidade de plantio para o estabel ecimento e para a produção de matéria seca de diversos adubos verdes (Fernandes et al., 1999; Amabile et al., 2000). Por outro lado, há carência de informações relativas às leguminosas herbáceas perenes. 
Os objetivos deste trabal ho foram determinar as taxas de cobertura do sol o e os acúmulos de matéria seca e N, P e K na parte aérea das leguminosas herbáceas perenes cudzu tropical e galáxia, considerando espaçamentos e densidades de plantio.

\section{MATERIAL E MÉTODOS}

O trabalho foi realizado na Embrapa Agrobiologia, Seropédica (RJ), num Argissolo Vermelho - Amarelo, cujos resultados da análise química $(0-20 \mathrm{~cm})$ de amostras de solo no início do experimento apresentaram os seguintes val ores: $\mathrm{pH}$ em $\mathrm{H}_{2} \mathrm{O}=(1: 2,5)=5,0 ; \mathrm{Al}=0,0 \mathrm{mmol}_{\mathrm{c}} \mathrm{dm}^{-3} ; \mathrm{Ca}=$ $32 \mathrm{mmol}_{\mathrm{C}} \mathrm{dm}^{-3} ; \mathrm{Mg}=13 \mathrm{mmol}_{\mathrm{C}} \mathrm{dm}^{-3} ; \mathrm{K}=73 \mathrm{mg} \mathrm{dm}^{-3}$ eP $=3 \mathrm{mg} \mathrm{dm}^{-3}$ de solo. Não foram aplicados cal cário nem fertilizantes para correção do sol o em razão da rusticidade das espécies estudadas.

O delineamento experimental foi blocos ao acaso, em arranjo fatorial $2 \times 2 \times 4$, com quatro repetições. Os tratamentos constaram de espécies (galáxia Galactia striata; e cudzu tropical - Pueraria phaseoloides, leguminosas de ciclo perene e hábito decrescimento ereto), espaçamentos entre sulcos de plantio (25 e $50 \mathrm{~cm}$ ) e densidades de plantas (5, 10, 15 e 20 plantas $\mathrm{m}^{-1}$ ).

As parcelas constaram de uma área de $4 \mathrm{~m}^{2}$ $(2 \times 2 \mathrm{~m})$. A semeadura foi realizada no período das águas (dezembro/98), pel osistema convencional , com uma aração e duas gradagens, a fim de reduzir a infestação de espontâneas da área, na qual predominava a tiririca (Cyperus rotundus). $\mathrm{Na}$ véspera da semeadura, as sementes sofreram escarificação térmica (imersão em água a $90^{\circ} \mathrm{C}$ por uma hora) e estirpes de bactérias do gênero Rhizobium recomendadas pela Embrapa Agrobiologia foram inoculadas nas sementes. Utilizaram-se altas densidades de semeadura, sendo, posteriormente, a população ajustada pelo desbaste manual. O controle das plantas espontâneas foi feito a partir de capinas manuais realizadas aos 26, 41 e 52 dias do plantio (DAP).

As avaliações constaram da taxa de cobertura do solo, determinada com auxílio do software SIARCS 3.0 (Sistema Integrado para Análise de Raízes e Cobertura do Solo), desenvolvido pela E mbrapa Instrumentação Agropecuária. O acompanhamento do crescimento das plantas, atéa cobertura total do solo, deu-se a partir de imagens fotográficas, tomadas aos 33, 43, 51, 61, 79, 99, 111 e 131 DAP. A câmara fotográfica era suspensa por um tripé, de modo queas fotografias fossem tomadas perpendicularmente ao solo, a uma altura de 1,60 m da superfície do terreno. Cada fotografia abrangia uma área de $1 \mathrm{~m}^{2}$ e foi tomada entre 7:30 e 9:00 h para atenuar efeitos adversos de sombra.
$O$ corte das plantas foi efetuado aos cinco meses do plantio (MAP) ( 1 - corte) e 12 MAP ( $2 \circ$ corte), a uma altura aproximada de $15 \mathrm{~cm}$ da superfície do solo com auxílio de tesoura de poda, permanecendo o material na superfície do terreno.

Quantificou-se a produção de matéria fresca de parte aérea pertencente à área útil de $1 \mathrm{~m}^{2}$, sendo, em seguida, retirada uma amostra de cada parcela, que foi pesada e colocada em estufa de ventilação forçada de ar a $65^{\circ} \mathrm{C}$ até atingir massa constante, para determinar a produção de matéria seca. O conteúdo de $\mathrm{N}$ foi determinado após digestão sulfúrica e destilação em Kjeldahl (Bremner \& Mulvaney, 1982); os conteúdos de $\mathrm{P}$ e K foram determinados após digestão nítrico-perclórica (Bataglia et al., 1983), sendo o P determinado em espectrofotômetro a partir de formação da cor azul do complexo fosfato-mol ibdato em meio sulfúrico, na presença de ácido ascórbico como redutor, e o K em espectrofotômetro de absorção atômica, por ocasião do primeiro edo segundo corte das plantas, realizados, respectiva-mente, aos 5 e 12 meses do plantio (MAP).

As análises estatísticas foram realizadas com auxílio doSAEG (Sistema para Análises Estatísticas e Genéticas), versão 5.0 (Euclydes, 1983), e constaram de análise de variância, aplicando-se o teste $\mathrm{F}$ para detectar significância a 5 e 1 \%. Para a taxa de cobertura do solo, as curvas foram ajustadas de acordo com a função logística $Y=A / 1+B e^{-K X}$, em que Y é a taxa de cobertura do solo (em \%); A é olimitesuperior da taxa de cobertura do solo; B está relacionado com o tamanho inicial do sistema; K éa taxa de incremento inerente do sistema, e X, dias após o plantio. Em relaçãoà matéria seca, aos teores e ao acúmulo de N, P e K, cujo teste F mostrou-se significativo, compararam-se as médias, utilizando o teste Tukey a $5 \%$.

\section{RESULTADOS E DISCUSSÃO}

A interpretação da análise de variância revelou efeitos significativos $(p \leq 0,05)$ de espaçamento, densidade e interação destes dois fatores na cobertura do solo. Dessa forma, curvas de taxa de cobertura do solo foram ajustadas pelo modelo de crescimento exponencial, considerando as combi nações entre os dois espaçamentos e as quatro densi dades de plantio para o cudzu tropical egaláxia. Para matéria seca e acúmulo de N, P e K, houve efeito significativo $(p \leq 0,05)$ de espécie, espaçamento e densidade apenas no primeiro corte das plantas, não ocorrendo, entretanto, interação entre fatores para estes mesmos parâmetros tanto no primeiro como no segundo corte.

$\mathrm{O}$ aumento do número de plantas $\mathrm{m}^{-1}$ não antecipou a completa cobertura do solo para as espécies em estudo. Contrariamente, a galáxia no espaçamento de $50 \mathrm{~cm}$ cobriu plenamente o sol o aos 
125 DAP, enquanto a diminuição desteespaçamento para $25 \mathrm{~cm}$ entre sulcos de plantio proporcionou redução de 14 dias para atingir a mesma cobertura do solo (Figura 1). Já o cudzu tropical no espaçamento de $50 \mathrm{~cm}$ proporcionou plena cobertura do terreno aos $123 \mathrm{DAP}$, ao passo que o menor espaçamento testado $(25 \mathrm{~cm})$ conferiu completa cobertura do solo aos 115 DAP (Figura 2). Comportamento semel hantetambém foi evidenciado por Guerra \& Teixeira (1997) para as leguminosas perenes; calopogônio, siratro, cudzu tropical e estilosantes e por Perin et al. (2000) para o amendoim forrageiro, siratro e centrosema, em condições edafocl imáticas semel hantes.

Embora tenha sido observado o efeito de espaçamento sobre a cobertura do solo, os resultados mostraram que o tempo necessário para a completa cobertura do solo pelas espécies avaliadas foi pouco expressivo. Comportamento similar também foi detectado por Perin et al. (2003) com o amendoim forrageiro, quando estabel ecidoa partir de diferentes densidades deplantio. Entretanto, diferenças marcantes ocorreram na fase inicial deestabelecimento para ambas as espécies, consi derada a fase crítica quanto à competição com a vegetação espontânea e susceptibilidade à erosão. Dessa forma, o estudo do arranjo populacional destas plantas, visando ao seu estabel ecimento, requer maior atenção atéa fase do $\mathrm{t}_{1 / 2}$ (tempo necessário para atingir $50 \%$ de cobertura), enão atéo período para a plena cobertura do terreno.

A partir das equações ajustadas, a galáxia no espaçamento $25 \mathrm{~cm}$ com 15 plantas $\mathrm{m}^{-1}$ apresentou $t_{1 / 2}$ de 52 DAP, enquanto no espaçamento $50 \mathrm{~cm}$ com

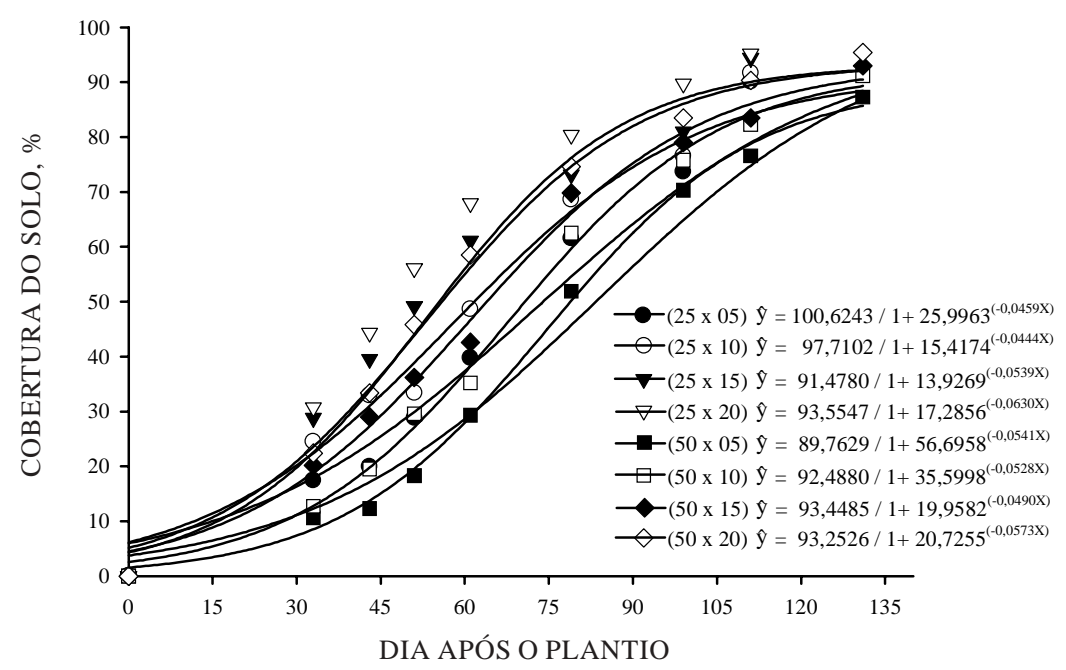

Figura 1. Taxas de cobertura do solo $(Y)$ por galáxia, considerando diferentes espaçamentos e densidades de plantio. Equações significativas a $1 \%$ pelo teste $F, \operatorname{com} R^{2}=0,99$.

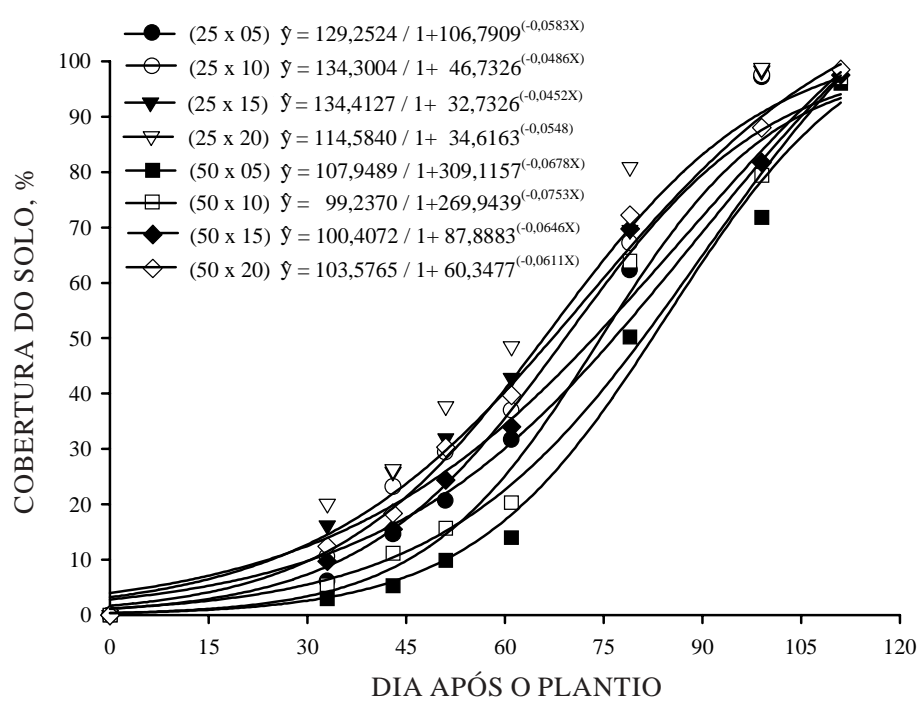

Figura 2. Taxas de cobertura do solo $(Y)$ por cudzu tropical, considerando diferentes espaçamentos e densidades de plantio. Equações significativas a $1 \%$ pelo teste $F$, com $R^{2}=0,99$. 
o mesmo número de plantas, o $\mathrm{t}_{1 / 2}$ foi atingido somente aos 64 DAP (Quadro 1). Para o cudzu tropical, o espaçamento $25 \mathrm{~cm}$ com 10 plantas $\mathrm{m}^{-1}$ apresentou $t_{1 / 2}$ aos 68 DAP, enquanto nesta mesma densidade com $50 \mathrm{~cm}$ entre sulcos, a mesma cobertura do solo foi atingida somente aos 74 DAP (Quadro 1). Esta diferença de 12 dias para a galáxia e seis dias para o cudzu tropical no $t_{1 / 2}$ é extremamente importante pelo fato de as plantas se encontrarem na fase inicial de estabel ecimento e de maior competição com a vegetação espontânea. Além disso, a rapidez na cobertura do sol o minimiza a erosão. Dechen et al. (1981) e Amado et al. (1987) demonstraram que o aumento da proporção deárea coberta reduz a erosão, enquanto Alvarenga et al. (1995) destacaram que uma cobertura uniforme de $20 \%$ do terreno é capaz de reduzir as perdas de solo em aproximadamente $50 \%$, quando comparada com solo descoberto.

Ao avaliar a produção de matéria seca e o acúmulo de nutrientes para os diferentes espaçamentos e densidades de plantas, notou-se, no primeiro corte, quea mai or produção de matéria seca eacúmulo de $\mathrm{N}, \mathrm{P}$ eK nas leguminosas foram obtidos no espaçamento de $25 \mathrm{~cm}$ (Quadro 2). Quanto ao efeito de densidade de plantio, a maior produção de matéria seca e os maiores acúmulos de $\mathrm{N}$ eK foram evidenciados nas densidades de 10, 15 e 20 plantas $\mathrm{m}^{-1}$ (Quadro 3). Acredita-se, com base nesses resultados, que o espaçamento de $25 \mathrm{~cm}$ na
Quadro 1. Tempo em dias após o plantio de cudzu tropical egaláxia para atingir $50 \%$ da cobertura do solo (t1/2), de acordo com os diferentes espaçamentos e densidades de plantio

\begin{tabular}{|c|c|c|c|}
\hline $\begin{array}{l}\text { E spaçamento } \\
\text { entre sulcos }\end{array}$ & Densidade & Cudzu tropical & Galáx \\
\hline $\mathrm{cm}$ & $\mathrm{pl} \mathrm{m}^{-1}$ & $-\mathrm{t}^{1} / 2(\mathrm{di}$ & a) \\
\hline 25 & 05 & 72 & 71 \\
\hline 25 & 10 & 68 & 62 \\
\hline 25 & 15 & 65 & 52 \\
\hline 25 & 20 & 60 & 47 \\
\hline 50 & 05 & 82 & 79 \\
\hline 50 & 10 & 74 & 71 \\
\hline 50 & 15 & 69 & 64 \\
\hline 50 & 20 & 66 & 55 \\
\hline
\end{tabular}

densidade 10 plantas $\mathrm{m}^{-1}$ possa ser o mais eficiente para a produção de matéria seca e para o acúmulo de nutrientes para a galáxia e cudzu tropical, por constituir a menor quantidade de sementes comparativamenteàs densidades de 15 e 20 plantas.

Os efeitos evidenciados no primei ro corte sobrea matéria seca e nutrientes não se repetiram no segundo corte das plantas. O hábito de crescimento volúvel das leguminosas (Perin, 2001) possibilitou ao longo de sete meses (período entre o primeiro eo segundo corte), efeito compensatório das menores

Quadro 2. Produção de matéria seca e acúmulo de nitrogênio, fósforo e potássio na parte aérea de cudzu tropical e galáxia, considerando diferentes espaçamentos por ocasião do primeiro corte das plantas

\begin{tabular}{|c|c|c|c|c|c|c|c|}
\hline $\begin{array}{l}\text { E spaçamento } \\
\text { entre sulcos }\end{array}$ & Matéria seca & & & & & & \\
\hline $\mathrm{cm}$ & t ha-1 & $\mathrm{g} \mathrm{kg}^{-1}$ & kg ha-1 & $\mathrm{g} \mathrm{kg}^{-1}$ & kg ha-1 & $\mathrm{g} \mathrm{kg}^{-1}$ & $\mathrm{~kg} \mathrm{ha} \mathrm{-}^{-1}$ \\
\hline $\begin{array}{l}25 \\
50\end{array}$ & $\begin{array}{l}3,43 \mathrm{~A}^{(1)} \\
2,84 \mathrm{~B}\end{array}$ & $\begin{array}{l}25,43 \mathrm{~A} \\
26,71 \mathrm{~A}\end{array}$ & $\begin{array}{l}87,22 \mathrm{~A} \\
75,86 \mathrm{~B}\end{array}$ & $\begin{array}{l}1,76 \mathrm{~A} \\
1,83 \mathrm{~A}\end{array}$ & $\begin{array}{l}6,04 \mathrm{~A} \\
5,20 \mathrm{~B}\end{array}$ & $\begin{array}{l}9,52 \mathrm{~A} \\
9,16 \mathrm{~A}\end{array}$ & $\begin{array}{l}32,65 \mathrm{~A} \\
26,01 \mathrm{~B}\end{array}$ \\
\hline C.V. (\%) & 23 & 13 & 25 & 17 & 37 & 21 & 33 \\
\hline
\end{tabular}

(1) Valores seguidos de letras iguais, dentro da coluna, não diferem entre si pelo teste Tukey $(\mathrm{p} \leq 0,05)$. Média das duas leguminosas.

Quadro 3. Produção de matéria seca, teor e acúmulo de nitrogênio, fósforo e potássio na parte aérea de cudzu tropical e galáxia, considerando diferentes densidades de plantio por ocasião do primeiro corte das plantas

\begin{tabular}{|c|c|c|c|c|c|c|c|}
\hline \multirow{2}{*}{$\begin{array}{l}\text { Densidade } \\
\text { planta } \mathrm{m}^{-1}\end{array}$} & \multirow{2}{*}{$\frac{\text { Matéria seca }}{\mathrm{t} \mathrm{ha}^{-1}}$} & \multicolumn{2}{|c|}{$\mathbf{N}$} & \multicolumn{2}{|c|}{$\mathbf{P}$} & \multicolumn{2}{|c|}{$\mathbf{K}$} \\
\hline & & $\mathrm{g} \mathrm{kg}^{-1}$ & $\mathrm{~kg} \mathrm{ha}^{-1}$ & $\mathrm{~g} \mathrm{~kg}^{-1}$ & kg ha-1 & $\mathrm{g} \mathrm{kg}^{-1}$ & $\mathrm{~kg} \mathrm{ha}^{-1}$ \\
\hline $\begin{array}{r}5 \\
10 \\
15 \\
20\end{array}$ & $\begin{array}{l}2,54 \mathrm{~B}^{(1)} \\
3,37 \mathrm{~A} \\
3,36 \mathrm{~A} \\
3,28 \mathrm{~A}\end{array}$ & $\begin{array}{l}26,61 \mathrm{~A} \\
26,79 \mathrm{~A} \\
24,72 \mathrm{~A} \\
26,15 \mathrm{~A}\end{array}$ & $\begin{array}{l}68,07 \mathrm{~B} \\
90,03 \mathrm{~A} \\
82,11 \mathrm{AB} \\
84,84 \mathrm{AB}\end{array}$ & $\begin{array}{l}1,90 \mathrm{~A} \\
1,72 \mathrm{~A} \\
1,77 \mathrm{~A} \\
1,77 \mathrm{~A}\end{array}$ & $\begin{array}{l}4,94 \mathrm{~A} \\
5,70 \mathrm{~A} \\
5,93 \mathrm{~A} \\
5,83 \mathrm{~A}\end{array}$ & $\begin{array}{l}9,09 \mathrm{~A} \\
9,12 \mathrm{~A} \\
9,65 \mathrm{~A} \\
9,48 \mathrm{~A}\end{array}$ & $\begin{array}{l}23,47 \mathrm{~B} \\
31,50 \mathrm{~A} \\
32,72 \mathrm{~A} \\
31,47 \mathrm{~A}\end{array}$ \\
\hline C.V. (\%) & 23 & 13 & 25 & 17 & 37 & 21 & 33 \\
\hline
\end{tabular}

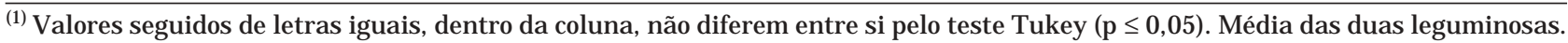


Quadro 4. Produção de matéria seca, teor e acúmulo de nitrogênio, fósforo e potássio na parte aérea de cudzu tropical e galáxia por ocasião do primeiro e do segundo corte das plantas

\begin{tabular}{|c|c|c|c|c|c|c|c|}
\hline \multirow[t]{2}{*}{ Espécie } & \multirow{2}{*}{$\frac{\text { Matéria seca }}{\mathrm{t} \mathrm{ha}^{-1}}$} & \multicolumn{2}{|c|}{$\mathbf{N}$} & \multicolumn{2}{|c|}{$\mathbf{P}$} & \multicolumn{2}{|c|}{$\mathbf{K}$} \\
\hline & & $\mathrm{g} \mathrm{kg}^{-1}$ & $\mathrm{~kg} \mathrm{ha}^{-1}$ & $\mathrm{~g} \mathrm{~kg}^{-1}$ & $\mathrm{~kg} \mathrm{ha}^{-1}$ & $\mathrm{~g} \mathrm{~kg}^{-1}$ & $\mathrm{~kg} \mathrm{ha}^{-1}$ \\
\hline & \multicolumn{7}{|c|}{ Primeiro corte } \\
\hline $\begin{array}{l}\text { Cudzu tropical } \\
\text { Galáxia }\end{array}$ & $\begin{array}{l}2,85 \mathrm{~B}^{(1)} \\
3,43 \mathrm{~A}\end{array}$ & $\begin{array}{l}25,97 \mathrm{~A} \\
26,17 \mathrm{~A}\end{array}$ & $\begin{array}{l}75,66 \mathrm{~B} \\
86,86 \mathrm{~A}\end{array}$ & $\begin{array}{l}1,76 \mathrm{~B} \\
1,83 \mathrm{~A}\end{array}$ & $\begin{array}{l}5,16 \mathrm{~B} \\
6,05 \mathrm{~A}\end{array}$ & $\begin{array}{l}9,16 \mathrm{~B} \\
9,52 \mathrm{~A}\end{array}$ & $\begin{array}{l}26,71 \mathrm{~B} \\
52,87 \mathrm{~A}\end{array}$ \\
\hline \multirow[t]{2}{*}{ C.V. (\%) } & 23 & 13 & 25 & 17 & 37 & 21 & 33 \\
\hline & \multicolumn{7}{|c|}{ Segundo corte } \\
\hline $\begin{array}{l}\text { Cudzu tropical } \\
\text { Galáxia }\end{array}$ & $\begin{array}{l}3,74 \mathrm{~B} \\
4,75 \mathrm{~A}\end{array}$ & $\begin{array}{l}31,19 \mathrm{~B} \\
35,48 \mathrm{~A}\end{array}$ & $\begin{array}{l}116,32 \mathrm{~B} \\
164,06 \mathrm{~A}\end{array}$ & $\begin{array}{l}2,06 \mathrm{~B} \\
2,34 \mathrm{~A}\end{array}$ & $\begin{array}{r}7,85 \mathrm{~B} \\
11,04 \mathrm{~A}\end{array}$ & $\begin{array}{l}12,82 \mathrm{~B} \\
16,80 \mathrm{~A}\end{array}$ & $\begin{array}{l}48,69 \mathrm{~B} \\
78,48 \mathrm{~A}\end{array}$ \\
\hline C.V. (\%) & 15 & 11 & 17 & 18 & 23 & 23 & 26 \\
\hline
\end{tabular}

${ }^{(1)}$ Valores seguidos de letras iguais, dentro da coluna, não diferem entre si pelo teste Tukey $(p \leq 0,05)$.

densidades no crescimento das plantas, indicando maior taxa de crescimento relativo. Esse comportamento mostra que as duas leguminosas se estabeleceram na área já aos 12 MAP.

Contrastando as duas espécies, a galáxia conferiu maior produção de matéria seca, teores e acúmulo de N, P eK do que o cudzu tropical, exceto o teor de $\mathrm{N}$ no primeiro corte (Quadro 4). Conquanto o teor deK da galáxia tenha diferido dos obtidos por Rangel (1984), o teor de $P$ apresentou-se semelhante. Contudo, tais respostas podem ter sido influenciadas pelo nível de fertilidade do solo ao qual as plantas estavam submetidas.

Ao longo do período experimental (12 meses), a galáxia acumulou 8,2 t ha-1 de matéria seca e 251, 17 e $131 \mathrm{~kg} \mathrm{ha}^{-1}$, respectivamente, de N, P e K; já o cudzu tropical acumulou $6,6 \mathrm{t} \mathrm{ha}^{-1}$ de matéria seca e 192, 13 e $75 \mathrm{~kg} \mathrm{ha}^{-1}$, respectivamente, de N, P eK. Em condições experimentais semelhantes, Espindola (2001) verificou que $86 \%$ do N presente no tecido vegetal destas leguminosas foi derivado do processo de fixação biológica de $N_{2}$ (FBN). Assim, pode-se estimar que a galáxia e o cudzu tropical, respectivamente, podem aportar, via FBN, 216 e $165 \mathrm{~kg} \mathrm{ha}^{-1}$ de $\mathrm{N}$. Além disso, o cudzu tropical confere maior agregação do sol o (Perin et al ., 2002a), atenua as oscilações térmicas e hídricas do solo (Perin, 2001) e promove maior crescimento de bananeiras e precocidade na col heita dos cachos, quando comparado com a aplicação de $90 \mathrm{~kg} \mathrm{ha}^{-1}$ de N mineral (Perin et al., 2002b). Destaca-se, então, o al to potencial destas leguminosas perenes como cobertura viva, representando uma estratégia para a auto-suficiência em $\mathrm{N}$ na nutrição defruteiras, por minimizar ou dispensar a utilização da adubação nitrogenada com fertilizantes sintéticos ou outras fontes.

A tomada de decisão sobre o adequado arranjo populacional à formação da cobertura do sol o requer a análise conjunta do custo com sementes ou mudas, operações de plantio e capinas, nível tecnológico e disponibilidade de mão-de-obra, além do potencial agronômico das plantas, tais como: a taxa de crescimento e o acúmulo de matéria seca e nutrientes. A partir das equações ajustadas, observou-se que o espaçamento de $25 \mathrm{~cm}$ com 10 plantas $\mathrm{m}^{-1}$ mostrou-se adequado para a rápida cobertura do solo para o cudzu tropical e galáxia.

\section{CONCLUSÕES}

1. Os espaçamentos e as densi dades de plantio de cudzu tropical e galáxia influenciaram as taxas de cobertura do solo, produção de matéria seca eacúmulo de N, P eK por ocasião do primei ro corte das plantas.

2. A galáxia apresentou maior potencial para produção de matéria seca e acúmulo de N, P eK do que o cudzu tropical.

3. O espaçamento de $25 \mathrm{~cm}$ na densidade 10 plantas $\mathrm{m}^{-1}$ conferiu rapidez na cobertura do solo, maior produção de matéria seca e acúmulo de N, P eK para o cudzu tropical e galáxia.

\section{LITE RATURA CITADA}

ALVARENGA, R.C.; COSTA, L.M.; MOURA FILHO, W. \& REGAZZI, A.J . Características de alguns adubos verdes de interesse para a conservação e recuperação de solos. Pesq. Agropec. Bras., 30:175-185, 1995.

AMABILE, R.F.; FANCELLI, A.L. \& CARVALHO, A.M. Comportamento de espécies de adubos verdes em diferentes épocas de semeadura e espaçamentos na região dos cerrados. Pesq. Agropec. Bras., 35:47-54, 2000.

AMADO, T.J .C.; ALMEIDA, E.X.; DALL'AGNOL, I. \& MATOS, A.T. Determinação da cobertura do solo por adubos verdes. Florianópol is, Empresa de Pesquisa Agropecuária de Santa Catarina, 1987. 6p. (EMPASC. Pesquisa em Andamento, 78) 
BATAGLIA, O.C.; FURLANI, A.M.C.; TEIXEIRA, J .P.F.; FURLANI, P.R. \& GALLO,J .R. Método deanálisequímica de plantas. B. Téc. Inst. Agron., 78:1-48, 1983.

BREMNER, J .M. \& MULVANEY, C.S. Nitrogen total. In: PAGE, A.L.; MILLER, R.A. \& KEENEY, D.R., eds. Methods of soil analysis. 2.ed. Madison, American Society of Agronomy, 1982. p.595-624. (Agronomy, 9)

DECHEN, S.C.F.; LOMBARDI NETO, F. \& CASTRO, O.M. Gramíneas eleguminosas eseus restos culturais no controle de erosão em Latossolo Roxo. R. Bras. Ci. Solo, 5:133-137, 1981.

DUDA, G.P.; GUERRA, J .G.M.; MONTEIRO, M.T.; DE-POLLI, H. \& TEIXEIRA, M.G. Perennial herbaceous legumes as live soil mulches and their effects on $\mathrm{C}, \mathrm{N}$ and $\mathrm{P}$ of the microbial biomass. Sci. Agric., 60:139-147, 2003.

ESPINDOLA, J.A.A. Avaliação de leguminosas herbáceas perenes usadas como cobertura viva de solo e seus efeitos sobre a produção da bananeira (Musa spp.). Seropédica, Universidade Federal Rural do Rio deJ aneiro, 2001. 144p. (Tese de Doutorado)

EUCLYDES, R.F. Manual de utilização do programa SAEG (Sistema para Análises Estatísticas e Genéticas). Viçosa, Universidade Federal de Viçosa, 1983. 59p.

FERNANDES, M.F.; BARRETO, A.C. \& EMÍDIO FILHO, J. Fitomassa de adubos verdes e controle de plantas daninhas em diferentes densidades populacionais de leguminosas. Pesq. Agropec. Bras., 34:1593-1600, 1999.

GUERRA, J .G.M. \& TEIXEIRA, M.G. Avaliação inicial de algumas leguminosas herbáceas perenes para utilização como cobertura viva permanente de solo. Seropédica, EMBRAPA - Agrobiologia, 1997. 7p. (Comunicado Técnico, 16)

KLEINHENZ, V.; SCHNITZLER, W.H. \& MIDMORE, D.J . Effects of legume live-mulch on crop performance, soil available nitrogen and crop $\mathrm{N}$ status in intensive tropical vegetable production. Biol. Agric. Hortic., 14:261-278, 1997.
LANINI, W.T.; PITTENGER, D.R.; GRAVES, W.L.; MUÑOZ, F.\& AGAMALIAN, H.S. Sub clovers as living mulches for managing weeds in vegetables. California Agric., 43:25-27, 1989.

PERIN, A. Desempenho de leguminosas herbáceas perenes com potencial de utilização para cobertura viva e seus efeitos sobre alguns atributos físicos do solo. Seropédica, Universidade Federal Rural do Rio de J aneiro, 2001. 105p. (Tese de Mestrado)

PERIN, A.; GUERRA, J .G.M. \& TEIXEIRA, M.G. Cobertura do solo e acumulação de nutrientes pelo amendoim forrageiro. Pesq. Agropec. Bras., 38:791-796, 2003.

PERIN, A.; GUERRA, J.G.M.;TEIXEIRA, M.G.; PEREIRA, M.G. \& FONTANA, A. Efeito da cobertura viva com leguminosas herbáceas perenes na agregação de um Argissolo. R. Bras. Ci. Solo, 26:713-720, 2002a.

PERIN, A.; LIMA, E.A.; ESPINDOLA, J .A.A.; GUERRA, J .G.M.; TEIXEIRA, M.G. \& BUSQUET, R.N.B. Contribuição da cobertura viva de sol o com leguminosas herbáceas perenes no 2 o ciclo de bananeiras cultivar nanicão. Seropédica, EMBRAPA - Agrobiologia, 2002b. 6p. (Comunicado Técnico, 53)

PERIN, A.; TEIXEIRA, M.G. \& GUERRA, J .G.M. Desempenho de al gumas leguminosas com potencial para utilização como cobertura viva permanente de solo. Agronomia, 34:38-43, 2000.

RANGEL, J .H.A. E feito residual de fontes de fósforo e ação da calagem na produção de matéria seca e composição química de cinco leguminosas forrageiras tropicais. Piracicaba, Escola Superior de Agronomia "Luiz de Queiroz", 1984. 155p. (Tese de Mestrado)

SARRANTONIO, M. Opportunities and challenges for the inclusion of soil-improving crops in vegetable production systems. HortScience, 27:754-758, 1992.

WILES, L.J .; WILLIAM, R.D.; CRABTREE, G.D. \& RADOSEVICH, S.R. Analyzing competition between a living mulch and a vegetable crop in an interplanting system. J . Am. Soc. Hortic. Sci., 114:1029-1034, 1989. 